\title{
Adenocarcinoma of Jejunal Duplication Cyst-Case Report and Review of Literature
}

\author{
U. V. Akshay Viswanath ${ }^{1} \cdot$ Noushif Medappil $^{2} \cdot$ Abishek Rajan $^{2} \cdot$ Sajeesh Sahadevan $^{2} \cdot$ N. Seetha Lekshmy ${ }^{2}$. \\ K. P. Kavitha ${ }^{3}$
}

Received: 6 May 2020 / Accepted: 12 May 2021 / Published online: 6 August 2021

(C) Indian Association of Surgical Oncology 2021

\begin{abstract}
Alimentary tract duplication cyst refers to congenital malformations that involve the mesenteric side of bowel and share a common wall or blood supply with the bowel. Duplication cyst occurs in any segment of the gastrointestinal tract from mouth to anus, but is more commonly seen in the mesentery of the ileo-colic region. Duplication cyst presents more frequently in children with abdominal pain or swelling. In adults, the diagnosis of duplication cyst is incidental and can present with complications like bleed, fistula formation, inflammatory mass, and rarely malignant transformation. English-language literature review has shown only three cases of malignancies reported so far associated with jejunal duplication cyst. A case of incidentally detected metastatic adenocarcinoma in a jejunal duplication cyst is being reported here, along with literature review of malignancies associated with small bowel duplication cyst.
\end{abstract}

Keywords Adenocarcinoma $\cdot$ Duplication cyst $\cdot$ Jejunal duplication

\section{Introduction}

Gastrointestinal malformations occurring in the early embryonal stages specifically abnormal canalization result in duplication cyst (DC) of the alimentary tract (AT) [1]. DC can occur at any level of the AT, but the most common location is the ileum, followed by the esophagus, jejunum, colon, stomach, and appendix. DC can be communicating or a non-communicating, cystic or tubular structure, lined by a mucosal membrane. Intestinal duplications may remain asymptomatic throughout life or could manifest clinically, depending on their size, specific relation to the bowel segment, or in case of communication with the intestinal lumen [2]. Malignancies are rare complications in intestinal duplications and are reported with DC of small bowel, colon, and

Noushif Medappil

noushif@gmail.com

1 Department of General Surgery, Aster Malabar Institute of Medical Sciences (MIMS), Calicut, India

2 Department of Surgical Gastroenterology and Liver Transplant, Aster Malabar Institute of Medical Sciences (MIMS), Calicut, Kerala 673016, India

3 Department of Pathology, Aster Malabar Institute of Medical Sciences (MIMS), Calicut, India rectum [3]. In this report, we present a case of an adenocarcinoma arising in a coincidentally found cystic duplication of the jejunum, and present a review of malignancies reported with small bowel DC in the English-language literature.

\section{Case Report}

A 31-year-old male was incidentally detected to have cystic swelling in the abdomen on ultrasound imaging performed as part of an annual health checkup. General physical and systemic examinations were unremarkable. His hemogram, liver function tests, and kidney function tests were normal. Contrast-enhanced computed tomography scan of the abdomen showed a fluid attenuation tract arising from the mid-jejunal segment with a blind end close to the porto-splenic confluence (Fig. 1). The proximal end of this tract had areas of dilatation and wall thickening. A possible diagnosis of jejunal DC was considered and the patient has suggested an elective diagnostic laparoscopy since he was asymptomatic. He underwent diagnostic laparoscopy after 6 weeks. A DC of about $6 \times 4 \mathrm{~cm}$ was noted $2 \mathrm{ft}$ distal to duodeno-jejunal flexure on the mesenteric side of bowel. The distal end of cyst was blind towards the root of mesentery. There was a tubular component of DC extending along the jejunum for $9 \mathrm{~cm}$ length. Rest of the peritoneal cavity and viscera 
Fig. 1 Axial (A) and coronal (B) computed tomography images showing duplication cyst of jejunum
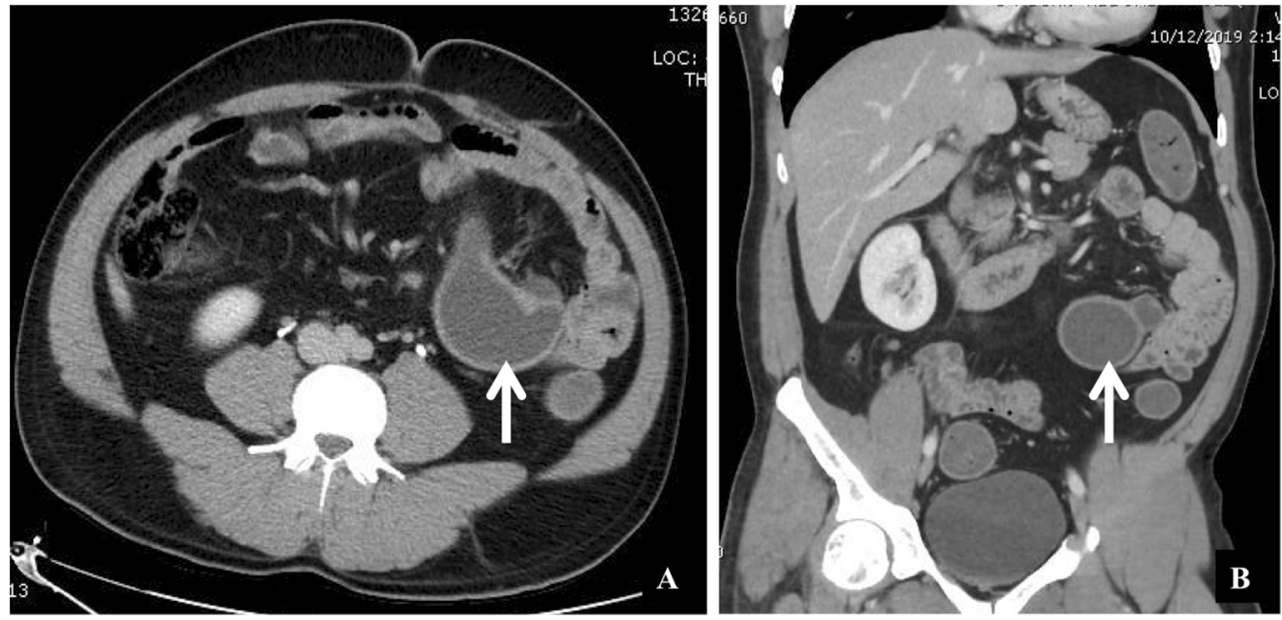

were normal on laparoscopy. The enteric DC with segment of jejunum was delivered through a mini-laparotomy. Segmental jejunal resection was performed and intestinal continuity restored by side-to-side jejuno-jejunostomy. The cyst wall had inflammatory thickening and contained cloudy odorless fluid. The patient had an uneventful postoperative recovery and was discharged on the sixth post-operative day.

Pathologic examination of the resected specimen (Fig. 2A) showed cyst wall mucosa lined by intestinal and gastric epithelium with severe dysplasia and tumor cells invading the muscle layer. The cells had moderate pleomorphic vesicular nuclei with distinct nucleoli (Fig. 2B-D). The histological impression was moderately differentiated adenocarcinoma arising from a jejunal DC. Two lymph nodes in the specimen were also positive for tumor. Further metastatic workup was performed with $18 \mathrm{~F}$-fluorodeoxyglucose positron emission tomography scan, which showed evidence of metastatic disease in the liver. He was referred for palliative chemotherapy in view of metastatic disease. Chemotherapy could not be initiated as patient had a travel abroad and restrictions imposed by Coronavirus (Covid-19) pandemic limited his further treatment. However, he had recurrent sub-acute adhesive intestinal obstruction and feed intolerance, which further delayed initiation of chemotherapy. He had an emergency reexploratory laparotomy after 2 months, which revealed adhesive jejunal obstruction with extensive omental and peritoneal deposits. A palliative jejuno-ileal bypass was performed, as the adhesions were extensive limiting a segmental resection.
Fig. 2 A-Segmental jejunal resection specimen showing the duplication cyst (arrow). B Histopathology showing mucosa lined by intestinal and gastric epithelium with severe dysplasia. C - Cells with moderately pleomorphic nuclei exhibiting distinct nucleoli. D-Tumor cells invading the muscle layer
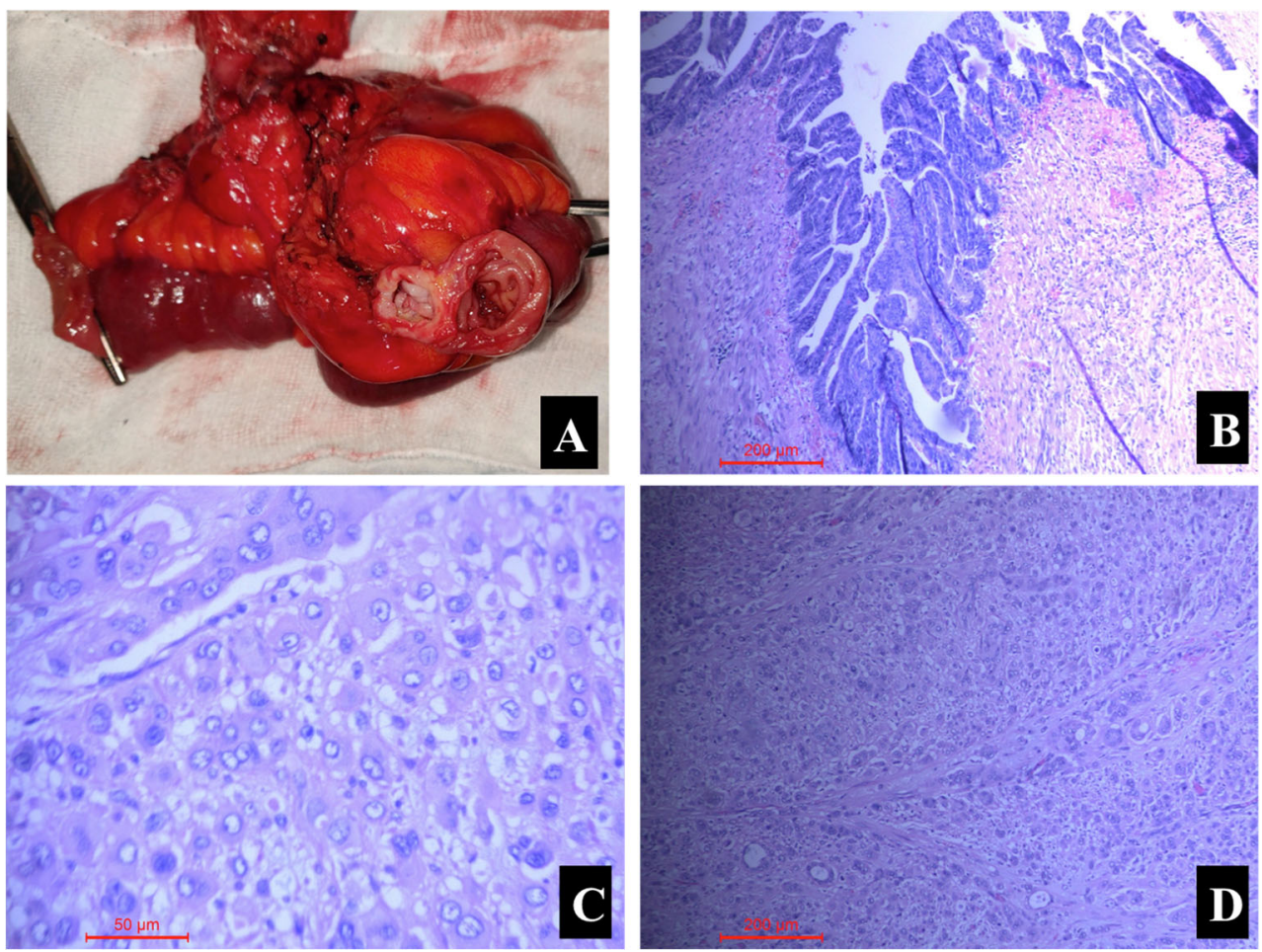


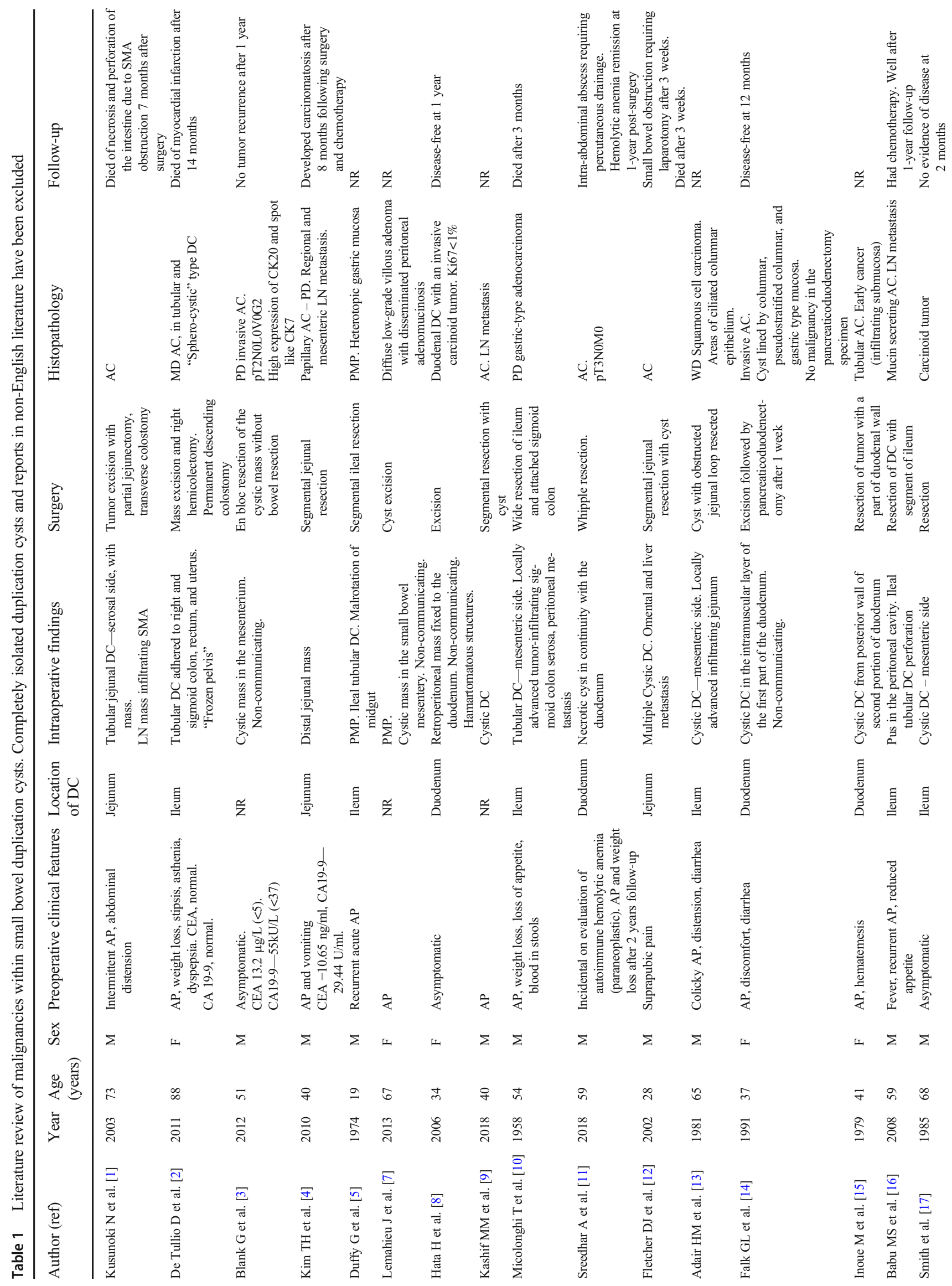


However, his general health deteriorated rapidly and he succumbed to sepsis after 3 weeks.

\section{Discussion}

Small bowel DC can be associated with all the three segments-duodenum, jejunum, and ileum, of which almost $50 \%$ of the duplications arise from jejunum. Various theories proposed regarding the development of DC include (a) recanalization after the solid epithelial stage of embryonic bowel development; (b) intrauterine vascular accident theory; (c) persistent embryologic diverticula, but no single theory can explain all the known duplications [2-4]. The accepted diagnostic criteria for an enteric DC include three characteristicsa well-developed smooth muscle coat, epithelial lining representing some portion of the AT mucosa, and intimate anatomic association with some portion of the AT [5]. However, cysts with no discernible communication or connection to the adjacent AT but with presence of typical histopathological features of DC would qualify for a diagnosis of an isolated DC [6]. The muscle within the wall of the DC and that of the adjacent bowel usually share a common blood supply; however, an isolated DC possesses its own exclusive blood supply [6].

Most DC are diagnosed during early childhood when they accompany symptoms such as abdominal pain or palpable mass. In contrast, these cysts are diagnosed incidentally during adulthood. Small bowel DC can be spherical cysts or tubular structures, located in or next to the mesenteric side of the bowel [7]. The lining mucosa of DC may not be necessarily that of the adjacent segment of the AT and can be derived from persistent mucosa in the embryonic stage when the duplication occurs. Complications arising from DC are attributed to the presence of heterotopic mucosa in these cysts. Ectopic gastric, pancreatic, squamous, transitional or ciliated mucosa, and ganglion cells can be found in the cyst wall. However, AT duplications are named according to the segment of the bowel in which they occur, rather than the features of their lining mucosa or hamartomatous change [8].

Various malignancies have been reported in enteric DC, including adenocarcinoma, squamous cell carcinoma, and carcinoid tumor [9]. Among the small bowel DC, malignant changes were most frequently reported with ileum. Carcinoma in jejunal duplication is extremely rare, and only three cases have been reported till date. The mode of growth and metastasis in these tumors is similar to that of primary small bowel cancer [4]. Preoperative diagnosis of tumors associated with DC is very difficult even after cross-sectional imaging with contrast-enhanced CT or MRI. Tumor markers (CEA, CA 19-9) may be helpful in preoperative detection of tumors [6]. Surgery is the treatment of choice for DC associated with malignancy. However, the delayed diagnosis from 
non-specific symptoms result in advanced stage of these tumors, thereby making the prognosis poor even after surgical resection $[1,9]$. A diagnosis of DC in an adult either before or at operation should have malignant change considered a possible reason for the onset of symptoms and radical excision undertaken where possible [10].

English-language literature on malignancies associated with small bowel DC was reviewed (Table 1). Three cases of adenocarcinoma associated with ileal DC reported in non-English literature were excluded. The patient's ages ranged from 19 to 88 years, and the male/female ratio of patients with malignant change in the DC of the small bowel was $2: 1$. The sites of DC were duodenum in 4 cases, jejunum in 4 cases, including the current case, ileum in 7 cases, and not specifically reported in 3 cases. Though abdominal pain was the most common symptom, 4 patients were asymptomatic at diagnosis of DC. One patient had paraneoplastic autoimmune hemolytic anemia leading to diagnosis of DC [11]. Tumor marker levels (CA19-9, CEA) were reported for three cases with adenocarcinoma of which two patients had elevated markers. Histologically, 13 patients had adenocarcinoma, two patients had carcinoid tumor, one patient had squamous cell carcinoma, and two patients had pseudomyxoma peritonei. Among the 13 patients with small bowel adenocarcinoma, 8 patients had metastatic disease at the time of surgery and 4 patients required aggressive resections including adjacent bowel or viscera. These suggest the aggressive nature of adenocarcinoma associated with small bowel DC.

In the present case, the enteric duplication had a cystic and tubular component. Tubular component was running parallel to the jejunal segment. Malignancy was detected in the DC only on histopathological examination. The adjacent bowel segment had neither precancerous lesions nor focus of malignancy. Tumor markers (CEA and CA19-9) were not done, as the diagnosis was late and staging evaluation later showed evidence of metastatic disease.

\section{Conclusion}

Malignant transformations within jejunal DC are rare and difficult to diagnose preoperatively. Intestinal DC in adults, though largely asymptomatic, should undergo immediate operative resection in view of unpredictable risk of malignancy.

Author Contributions All authors were involved in the clinical care of the patient. All authors have read and approved the manuscript.

\section{Declarations}

Competing Interests The authors declare no competing interests.

\section{References}

1. Kusunoki N, Shimada Y, Fukumoto S, Iwatani Y, Ohshima T, Arahi E, Miyazaki N, Maeda S (2003) Adenocarcinoma arising in a tubular duplication of the jejunum. J Gastroenterol 38(8):781-785

2. de Tullio D, Rinaldi R, Pellegrini D, Stano R, Messina F, Cavazzini L, Azzena G, Occhionorelli S (2011) Adenocarcinoma arising in an elderly patient's large ileal duplication. Int J Surg Pathol 19(5):681684

3. Blank G, Königsrainer A, Sipos B, Ladurner R (2012) Adenocarcinoma arising in a cystic duplication of the small bowel: case report and review of literature. World J Surg Oncol 10:55

4. Kim TH, Kim JK, Jang EH, Lee JH, Kim YB (2010) Papillary adenocarcinoma arising in a tubular duplication of the jejunum. Brit J Radiol 83(987):e61-e64

5. Duffy G, Enriquez AA, Watson WC (1974) Duplication of the ileum with heterotopic gastric mucosa pseudomyxoma peritonei and nonrotation of the midgut. Gastroenterology 67(2):341-346

6. Shin SY, Cho MY, Ryu H, Kim JW, Kim HS, Kim JM, Ko AN, Yu TS, Park HJ (2014) Adenocarcinoma originating from a completely isolated duplication cyst of the mesentery in an adult. Intest Res 12(4):328-332

7. Lemahieu J, D'Hoore A, Deloose S, Sciot R, Moerman P (2013) Pseudomyxoma peritonei originating from an intestinal duplication. Case Rep Pathol 608016

8. Hata H, Hiraoka N, Ojima H, Shimada K, Kosuge T, Shimoda T (2006) Carcinoid tumor arising in a duplication cyst of the duodenum. Pathol Int 56(5):272-278

9. Kashif MM, Satishrao I, Sachin DV (2018) Adenocarcinoma arising in a small bowel duplication cyst. J Med Sci Res 6(2):59-62

10. Micolonghi T, Meissner GF (1958) Gastric-type carcinoma arising in duplication of the small intestine. Ann Surg 147(1):124-127

11. Sreedhar A, Nair R, Scialla W (2018) Paraneoplastic auto-immune hemolytic anemia: an unusual sequela of enteric duplication cyst. Anticancer Res 38(1):509-512

12. Fletcher DJ, Goodfellow PB, Bardsley D (2002) Metastatic adenocarcinoma arising from a small bowel duplication cyst. Eur J Surg Oncol 28(1):93-94

13. Adair HM, Trowell JE (1981) Squamous cell carcinoma arising in a duplication of the small bowel. J Pathol 133(1):25-31

14. Falk GL, Young CJ, Parer J (1991) Adenocarcinoma arising in a duodenal duplication cyst: a case report. Aust N Z J Surg 61(7): $551-553$

15. Inoue M, Nishimura O, Andachi H, Koga S (1979) Early cancer of duodenal duplication. A case report. Gastroenterol Jpn 14(3):233237

16. Babu MS, Raza M (2008) Adenocarcinoma in an ileal duplication. J Assoc Physicians India 56:119-120

17. Smith JH, Hope PG (1985) Carcinoid tumor arising in a cystic duplication of the small bowel. Arch Pathol Lab Med 109(1):95-96

18. Tew K, Soans BK, Millar EA (2000) Adenocarcinoma in an ileal duplication cyst: ultrasound and computed tomography findings. Australas Radiol 44(2):228-231

Publisher's Note Springer Nature remains neutral with regard to jurisdictional claims in published maps and institutional affiliations. 